\title{
AN ORTHONORMAL SYSTEM ON THE CONSTRUCTION OF THE GENERALIZED CANTOR SET
}

RAAFAT RIAD RIZKALLA

\author{
Mathematics Department, Faculty of Education \\ Ain Shams University, Heliopolis, Cairo, Egypt \\ (Received January 16, 1992 and in revised form June 22, 1992)
}

ABSTRACT. This paper presents a new complete orthonormal system of functions defined on the interval $[0,1]$ and whose supports shrink to nothing. This system related to the construction of the Cantor ternary set. We defined the canonical map $\xi$ and proved the equivalence between this system and the Walsh system. The generalized Cantor set with any dissection ratio is established and the constructed system is defined in the general case.

KEY YORDS AND PHRASES. Dyadic expansion, Walsh functions, Cantor set. 1992 AMS SUBJECT CLASSIFICATION CODE. $42 \mathrm{C} 10$

\section{INTRODUCTION.}

We shall denote the set of positive integers by $P$, the set of non-negative integers by $N$, the set of real numbers by $R$, and the set of dyadic rationals in the unit interval $[0,1)$ by $Q$. In particular, each element of $Q$ has the form $p / 2^{n}$ for some $p, n \in N, 0 \leq p<2^{n}$. Each $n \in N$ has a unique dyadic expansion

$$
n=\sum_{k=0}^{\infty} n_{k} 2^{k} \quad, \quad n_{k} \in\{0,1\}
$$

where $n_{k}$ are called the dyadic coefficients of $n$. Likewise each $x \in[0,1)$ has a (unique) dyadic expansion

$$
x=\sum_{k=0}^{\infty} x_{k} 2^{-(k+1)}, \quad x_{k} \in\{0,1\}
$$

the finite expansion being chosen in case $x$ belongs to the dyadic rationals $Q$. In terms of the dyadic expansions, the dyadic sum of two numbers $x, y \in(0,1)$ is defined by

$$
x \stackrel{\circ}{+}=\sum_{k=0}^{\infty}\left|x_{k}-y_{k}\right| 2^{-(k+1)}
$$

The dyadic sum of a pair of integers $n, m \in N$ is defined by

$$
n \oplus m=\sum_{k=0}^{\infty}\left|n_{k}-m_{k}\right| 2^{-k}
$$

where $\left(n_{k}, k \in N\right)$ and $\left(m_{k}, k \in N\right)$ are the dyadic coefficients of $n$ and $m$.

Let $r$ be the function defined on $[0,1)$ by

$$
r(x)= \begin{cases}1 & x \in\left(0, \frac{1}{2}\right) \\ -1 & x \in\left[\frac{1}{2}, 1\right)\end{cases}
$$

extended to $R$ by periodicity of period 1 . The Rademacher system $\left(r_{n}, n \in N\right)$ is 
defined by, $r_{n}(x)=r\left(2^{n} x\right), x \in R, n \in N$. The first three Rademacher functions are shown in Figure 1.

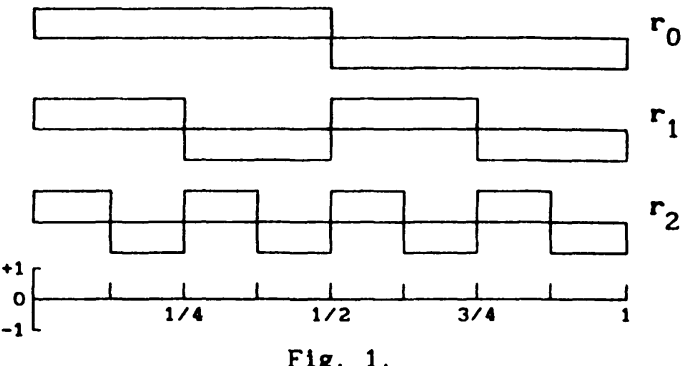

F1g. 1 .

The Rademacher functions form an incomplete set of orthonormal functions on $[0,1)$. The Walsh system ( $\left.w_{n}, n \in N\right)$ was introduced by Paley [11] in 1932 as products of Rademacher functions in the following way. If $n \in N$ has dyadic coefficients $(n, k \in N)$ then

$$
w_{n}=\prod_{k=0}^{\infty} r_{k}^{n_{k}}
$$

Walsh functions belong to the class of piecewise constant basis functions that have been developed in the twentieth century and have played an important role in scientific applications. The foundations of the Walsh functions field were made by Rademacher [12], Walsh [16], Fine [10], and Paley [11]. Owing to their salient properties, Walsh functions proved to be very powerful in solving various problems such as the analysis of dynamic systems [4-8], the design of optimal controllers [3], and the identification problem of dynamic systems [2],[14]. The first eight Walsh functions are shown in Figure 2 .

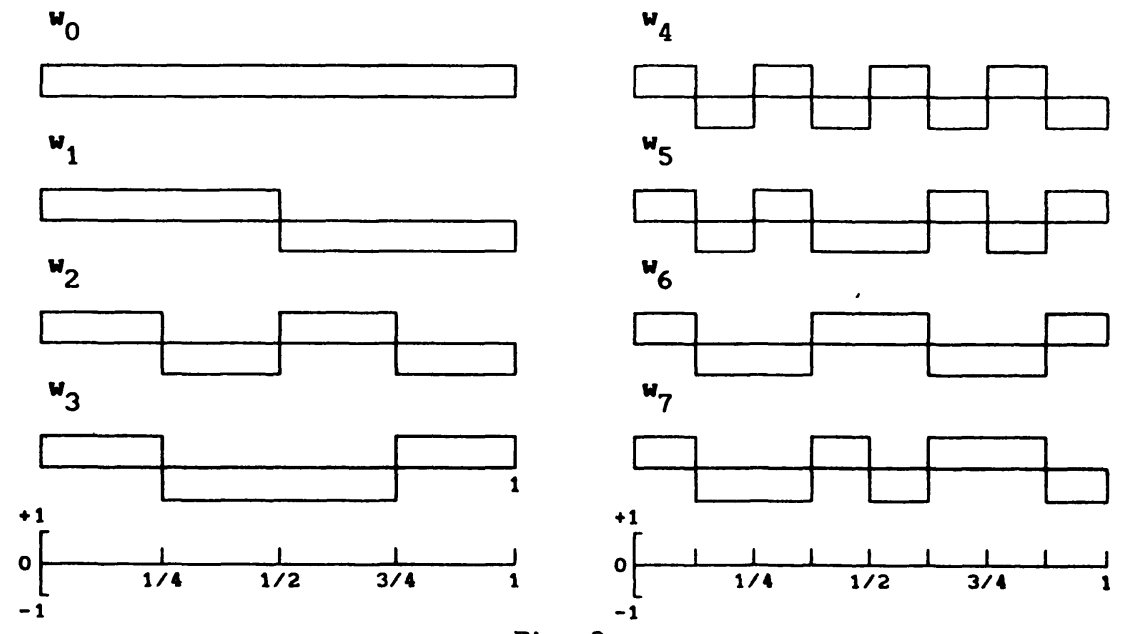

Fig. 2 .

Notice by definition that $w_{0}=1, w_{2^{n}}=r_{n} \quad$ for $n \in N$, and each Walsh function is piecewise constant with finitely many jump discontinuities on $(0,1)$ and takes only the values +1 or -1 . The Walsh system is orthonormal on $[0,1)$ and possess the properties (see [13]),

$$
\begin{aligned}
& w_{n} \cdot w_{m}=w_{n \oplus m} \\
& w_{n}(x+y)=w_{n}(x) w_{n}(y)
\end{aligned}
$$


By a dyadic interval in $[0,1)$ we shall mean an interval of the form

$$
I(p, n)=\left[\frac{p}{2^{n}}, \frac{p+1}{2^{n}}\right) \quad\left(0 \leq p<2^{n}, n, p \in N\right)
$$

For each $x \in[0,1)$ and $n \in N$ we shall denote the dyadic interval of length $2^{-n}$ which contains $x$ by $I_{n}(x)=I(p, n)$ where $0 \leq p<2^{n}$ is uniquely determined by the relationship $x \in I(p, n)$. The Walsh functions, being a complete orthonormal system (see [1],[16]) allows a representation of every absolutely integrable function $f(x)$ on $[0,1)$ in Walsh series in the form

$$
f(x)=\sum_{n=0}^{\infty} a_{n} w_{n}(x) \quad \text { where } \quad a_{n}=\int_{0}^{1} f(x) w_{n}(x) d x
$$

being the Walsh-Fourler coefficients of $f$. The $n$-th partial sum of Walsh series and the Dirichlet kernel are denoted respectively by [15]

$$
\mathrm{S}_{\mathrm{n}} \mathrm{f}=\sum_{\mathrm{k}=0}^{\mathrm{n}-1} \mathrm{a}_{\mathrm{k}} w_{k} \quad \text { and } \quad \mathrm{D}_{\mathrm{n}}=\sum_{k=0}^{n-1} w_{k}
$$

Fine [10], has shown that $S_{n} f(x)=\int_{0}^{1} f(t) D_{n}(x+y) d t$. The Dirichlet kernel satisfies $D_{2^{n}}(x)=2^{n}$ for $x \in I_{n}(0)=\left[0,2^{-n}\right)$ and zero elsewhere.

\section{THE CANTOR SET AND THE CANTOR FUNCTION}

The Cantor ternary set $C$ formed by removing middle thirds from the interval $[0,1]$ can be defined as follows: for each $n \in N$, construct the closed intervals

$$
J_{2^{n}+k}=\left[\frac{2 k}{3^{n}}, \frac{2 k+1}{3^{n}}\right] \quad, 0 \leq k<2^{n}
$$

where $k$ is defined by

$$
k^{*}=\sum_{i=0}^{\infty} k_{1} 3^{1}
$$

$k_{1}$ being the dyadic coefficients of $k$ in (1.1). Then the Cantor ternary set ( of ratio $1 / 3$ ) or more briefly the Cantor set C is

$$
C=\bigcap_{n=0}^{\infty} \bigcup_{k=0}^{2^{n}-1} J_{2^{n}+k}
$$

The middle open intervals removed in the above construction are defined by

$$
E=\bigcup_{n=0}^{\infty} \bigcup_{k=0}^{2^{n}-1} E_{2^{n}+k} \text { where } E_{2^{n}+k}=\left(\frac{6 k^{*}+1}{3^{n+1}}, \frac{6 k^{*}+2}{3^{n+1}}\right)
$$

Arithmetically, the Cantor set consists exactly of those points which can be expanded in the ternary system without using the digit 1 , i.e. for every $x \in C$

$$
x=\sum_{k=0}^{\infty} x_{k} \cdot 3^{-(k+1)}, \quad x_{k} \in\{0,2\}
$$

We define a class of closed intervals

$$
A_{n}=\left\{J_{2^{n}+k}: 0 \leq k<2^{n}\right\} \quad, n \in N
$$

For each $n \in N, A_{n}$ contains $2^{n}$ closed interval each of length $3^{-n}$. The Cantor set can be associated with a monotone non-decreasing continuous function called the Cantor - Lebesgue function and defined by the following process. For each closed interval $I$, let

$$
R_{I}(x)=\int_{0}^{x} \frac{x_{I}(t)}{|I|} d t \quad \text { where } \quad x_{I}(t)= \begin{cases}1 & t \in I \\ 0 & t \notin I\end{cases}
$$

$x_{I}$ and $\mid$ I $\mid$ represents the characteristic function and the length of the 
interval 1 , respectively. For each $n \in N$, let

Since

$$
F_{n}(x)=2^{-n} \sum_{k=0}^{2^{n}-1} R_{J_{2 n}^{n}+k}(x)
$$

$$
\begin{aligned}
R_{J_{2}{ }^{n}+k}(x) & =\int_{0}^{x} \frac{J_{2^{n}+k}}{\left|J_{2^{n}+k}\right|} d t=3^{n} \int_{0}^{x} x_{J_{2}^{n}+k}(t) d t \\
& = \begin{cases}0 & x<2 k^{*} \cdot 3^{-n} \\
3^{n} x-2 k & x \in J_{2}^{n}+k \\
1 & x>\left(2 k^{*}+1\right) \cdot 3^{-n}\end{cases}
\end{aligned}
$$

Hence, from (2.4), $F_{0}(x)=x \quad, x \in J_{1}=[0,1]$, and for $n \in P$

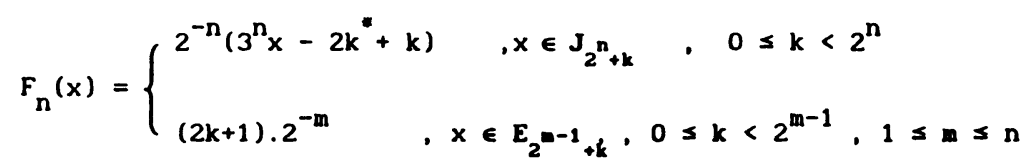
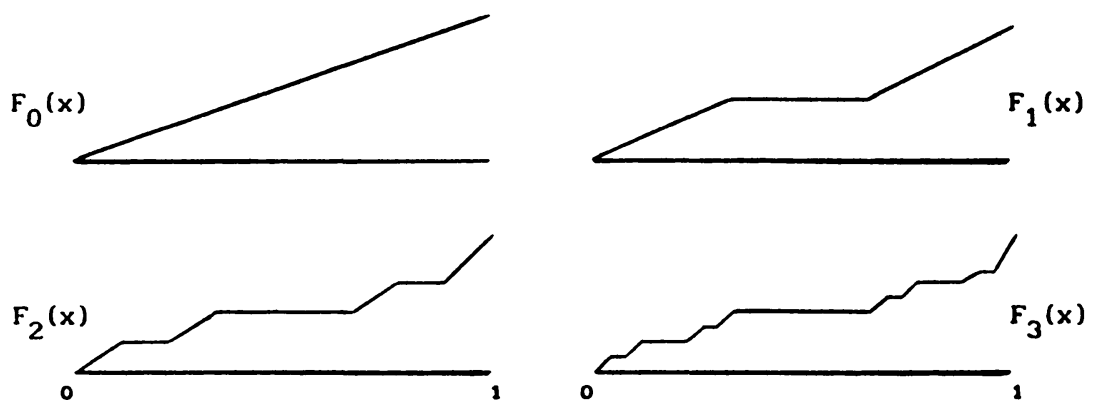

F1g.3.

We observe that, for each $n \in N$, the function $F_{n}(x)$ is continuous, nondecreasing on $[0,1]$, and satisfies $F_{n}(0)=0, F_{n}(1)=1$ (see figure 3 ). Indeed,

$$
\lim _{n \rightarrow \infty} F_{n}(x)=\frac{2 k+1}{2^{m}}, x \in E_{2^{m-1}+k}, 0 \leq k<2^{m-1}, 1 \leq n<\infty
$$

It is easy to see that $F(x)=\lim _{n \rightarrow \infty} F_{n}(x), x \in[0,1]$ exists, is continuous, monotone non-decreasing and satisfies $F(0)=0, F(1)=1$. This function is called the Cantor-Lebesgue function associated with the Cantor set $C$.

Now, we introduce the mapping $\xi: C \longrightarrow(0,1)$

$$
\xi(x)=\sum_{k=0}^{\infty} \bar{x}_{k} \cdot 2^{-(k+1)} \quad, x \in C
$$

where

$$
\bar{x}_{k}= \begin{cases}0 & \text { if } x_{k}=0 \\ 1 & \text { if } x_{k}=2,\end{cases}
$$

$x_{k}$ being the coefficients in the ternary expansion of $x$ in (2.3). The mapping $\xi$ satisfies the following properties:

$1-\xi$ is one-to-one mapping from $C / C^{*}$ onto $[0,1)$ where $C^{*}$ is a countable set contains all $x \in C$ which has ternary expansion terminates in 2 's. In fact.

$$
C^{*}=\left\{\frac{2 k^{*}+1}{3^{n}}, 0 \leq k<2^{n}, n \in N\right\}
$$


2- For each $n \in N, 0 \leq k<2^{n} \quad \xi\left(\frac{2 k}{3^{n}}\right)=\frac{k}{2^{n}} \quad$ and

$$
x \in\left(C / C^{*}\right) \cap J_{2^{n}+k} \text { iff } \xi(x) \in I_{n}\left(\frac{k}{2^{n}}\right) \text {. }
$$

where $I_{n}(y)$ is the dyadic interval of length $2^{-n}$ which contains $y$.

Define the Lebesgue-stieltjes measure $\mu_{F}(\alpha, \beta)=F(\beta)-F(\alpha)$ where $F$ is the Cantor function. Indeed, for each $0 \leq k<2^{n}, n \in N$

3. AN ORTHONORMAL SYSTEM OF FUNCTIONS

$$
\mu_{F}\left(J_{2^{n}+k}\right)=2^{-n}=\left|I_{n}\left(\frac{k}{2^{n}}\right)\right|
$$

We construct a function system $\Phi=\left\{\varphi_{n}: n \in N\right\}$ on the interval [0,1) in the following way: for each $x \in[0,1)$ and $n \in N, 0 \leq k<2^{n+1}$, i.e. $k=\sum_{i=0}^{n} k_{1} 2^{i}$, define

$$
\varphi_{n}(x)= \begin{cases}(-1)^{k_{0}} & x \in J_{2} n+1+k \\ 0 & \text { otherwise }\end{cases}
$$

It is obvious that $\varphi_{n}$ has the constant value +1 on half of the closed intervals in $A_{n+1}$ while on the other half $\varphi_{n}$ has constant value -1 . Indeed, $\varphi_{n}$ is odd function about $t=1 / 2$ (see figure 4 ). Consequently,

$$
\int_{0}^{1} \varphi_{n} d \mu_{F}=0 \quad, n \in N
$$

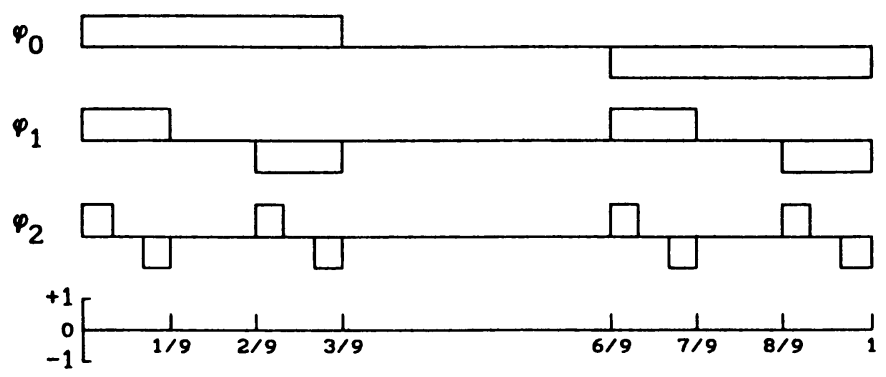

Fig. 4.

THEOREM 1. The system $\left\{\varphi_{n}: n \in N\right\}$ is orthonormal with respect to the measure $\mu_{\mathrm{F}}$, 1.e.

$$
\int_{0}^{1} \varphi_{\mathrm{n}} \varphi_{\mathrm{m}} \mathrm{d} \mu_{\mathrm{F}}= \begin{cases}0 & , \mathrm{n} \neq \mathrm{m} \\ 1 & , \mathrm{n}=\mathrm{m} .\end{cases}
$$

PROOF. Let $n>m \geq 0$ and $J \in A_{m+1}$. Since $\varphi_{m}$ has constant value +1 or -1 on $J$, and $J$ contains an even number (namely $2^{n-m}$ ) of closed intervals in $A_{m+1}$; on half of these intervals $\varphi_{n}$ has the constant value +1 while on the other half $\varphi_{n}$ has constant value -1 , consequently,

$$
\int \varphi_{\mathrm{n}} \varphi_{\mathrm{m}} \mathrm{d} \mu_{\mathrm{F}}= \pm \int_{\mathrm{J}} \varphi_{\mathrm{n}} \mathrm{d} \mu_{\mathrm{F}}=0 .
$$

Summing over all $J \in A_{m+1}$ we get $\int_{0}^{1} \varphi_{n} \varphi_{m} d \mu_{F}=0$. If $n=m$, then

$$
\int_{0}^{1} \varphi_{n}^{2} d \mu_{F}=\sum_{J \in A_{n+1}} \int d \mu_{F}=\sum_{J \in A_{n+1}} \mu_{F}^{(J)}
$$

Since $\quad \mu_{F}(J)=2^{-(n+1)} \quad \forall J \in A_{n+1}, n \in N$ and $A_{n+1}$ contains $2^{n+1}$ closed interval, then

$$
\int_{0}^{1} \varphi_{n}^{2} d \mu_{F}=1 \quad, n \in N
$$


COROLlary 1. For each $x \in \mathbf{C} / \mathbf{C}^{*}, n \in N$

$$
\varphi_{n}(x)=(-1)^{\bar{x}_{n}}=\left(r_{n} \circ \xi\right)(x)
$$

where $\left(r_{n}, n \in N\right)$ be the Rademacher system.

PRoOF. Let $n \in N, 0 \leq k<2^{n+1}$ and $x \in\left(C / C^{*}\right) \cap J_{2^{n+1}+k}$. From the properties of the mapping $\xi$ we have $\xi(x) \in I_{n+1}\left(\frac{k}{2^{n+1}}\right), \bar{x}_{n}=k_{0}$. Since

then

$$
\varphi_{n}(x)=(-1)^{k_{0}}, x \in J_{2}^{n+1}+k \text { and }\left(r_{n} \circ \xi\right)(x)=r_{n}(\xi(x))=(-1)^{\bar{x}_{n}}
$$

$$
\varphi_{n}(x)=(-1)^{\bar{x}_{n}}=\left(r_{n} \circ \xi\right)(x) \quad, \quad x \in C / C, n \in N .
$$

Now, we define the product system $\Psi=\left\{\psi_{n}, n \in N\right\}$ by

$$
\psi_{n}=\prod_{k=0}^{\infty} \varphi_{k}^{n} \quad, \quad n \in N
$$

where $\left(n_{k}, k \in N\right)$ are the dyadic coefficients of $n$.

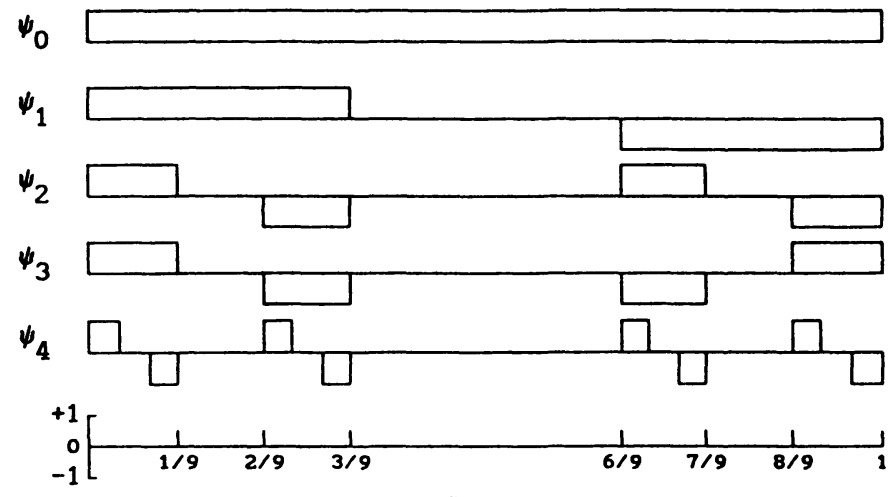

Fig. 5.

The function system $\Psi$ satisfies the following properties:

1. For each $n, m \in N, \quad \psi_{n} \cdot \psi_{m}=\psi_{n \rightarrow m} \quad$ where $\oplus$ is the dyadic sum.

2. For each $n, m \in N, m=2^{n}+k, 0<k<2^{n}, \psi_{m}$ is even function with respect to $\frac{1}{2}$ and $\psi_{2^{n}}=\varphi_{n}$ is odd function with respect to $\frac{1}{2}$ (see figure 5 ).

3. For each $n, m \in N, 2^{n} \leq m<2^{n+1}, \psi_{m}$ has constant value +1 on half of the closed intervals in $\alpha_{n+1}$ while on the other half $\psi_{m}$ has constant value -1 . consequently,

In general,

$$
\int_{0}^{1} \psi_{m} d \mu_{F}=0, \quad 2^{n} \leq m<2^{n+1}, n \in N
$$

$$
\int_{0}^{1} \psi_{n} d \mu_{F}=0 \quad, n \in P
$$

4. Let $n \in N$ with dyadic coefficients $\left(n_{k}, k \in N\right)$ and $x \in C / C^{*}$. From equations (3.3), (3.4), we have

where

$$
\psi_{n}(x)=\prod_{k=0}^{\infty}(-1)^{n_{k} \bar{x}_{k}}=(-1)^{\langle n, \xi(x)\rangle}
$$

$$
\langle n, \xi(x)\rangle=\sum_{k=0}^{\infty} n_{k} \bar{x}_{k} \quad(\bmod 2)
$$


COROLLARY 2. For each $x \in \mathrm{C} / \mathrm{C}^{*}, \mathrm{n} \in \mathrm{N}$

$$
\psi_{n}(x)=\left(w_{n} \circ \xi\right)(x)
$$

where ( $\left.w_{n}, n \in N\right)$ be the Walsh system.

PROOF. From equations (1.5), (3.3) and (3.4), we have

$$
\begin{aligned}
\psi_{n}(x) & =\prod_{k=0}^{\infty}\left(\varphi_{k}(x)\right)^{n_{k}}=\prod_{k=0}^{\infty}\left(\left(r_{k} \circ \xi\right)(x)\right)^{n_{k}} \\
& =\prod_{k=0}^{\infty}\left(r_{k}(\xi(x))\right)^{n_{k}}=w_{n}(\xi(x)) \\
& =\left(w_{n} \circ \xi\right)(x) .
\end{aligned}
$$

According to corollary 2 and the properties of the mapping $\xi$, it is obvious that

$$
\psi_{n}\left(\frac{2 k}{3^{n}}\right)=w_{n}\left(\frac{k}{2^{n}}\right) \quad, 0 \leq k<2^{n}, n \in N
$$

THEORDM 2. The system $\left\{\psi_{n}, n \in N\right\}$ is orthonormal with respect to the measure $\mu_{F}, 1 . e$.

$$
\int_{0}^{1} \psi_{1} \psi_{j} d \mu_{F}= \begin{cases}0 & , 1 \neq j \\ 1 & , 1=j\end{cases}
$$

PROOF. First, let $i \neq j$. Since $\psi_{i} \cdot \psi_{j}=\psi_{1 \oplus j}$, it suffices to show that $\int_{0}^{1} \psi_{m} d \mu_{F}=0, \quad m \in N$, which is clear from (3.5). Second, in case $1=j$, let $n, m \in N, 2^{n} \leq m<2^{n+1}$, then

$$
\int_{0}^{1} \psi_{m}^{2} d \mu_{F}=\sum_{J \in d} \int_{J+1} d \mu_{F}=\sum_{J \in A_{n+1}} \mu_{F}(J)=2^{n+1} \cdot 2^{-(n+1)}=1
$$

THEOREM 3. The system $\Psi=\left\{\psi_{n}, n \in N\right\}$ is complete, with respect to the measure $\mu_{F}$, on the Cantor set $C$.

PRoor. Let $n, m \in N, 0 \leq m<2^{n}$ and suppose that $f$ is integrable function for which

$$
\int_{0}^{1} f \psi_{m} d \mu_{F}=0 \quad, 0 \leq m<2^{n}
$$

Since each function $\psi, 0 \leq m<2^{n}$, has only one fixed value on each interval $J_{2} n_{k}$. Let

Then (3.9) gives,

$$
\Gamma_{n}^{(k)}=\int_{J_{2}+k} f d \mu_{F}=\int_{k \cdot 3^{-n}}^{(2 k+1) \cdot 3^{-n}} f d \mu_{F}, 0 \leq k<2^{n} .
$$

$$
\int_{0}^{1} f \psi_{m} d \mu_{F}=\sum_{k=0}^{2^{n}-1} \Gamma_{n}^{(k)} \psi_{m}\left(\frac{2 k}{3^{n}}\right)=0,0 \leq m<2^{n}
$$

From (3.7), we get

$$
\psi_{m}\left(\frac{2 k}{3^{n}}\right)=w_{m}\left(\frac{k}{2^{n}}\right), \quad 0 \leq k<2^{n}, \quad 0 \leq m<2^{n}, \quad n \in N,
$$

then

$$
\sum_{k=0}^{2^{n}-1} \Gamma_{n}^{(k)} w_{m}\left(\frac{k}{2^{n}}\right)=0 \quad, 0 \leq m<2^{n}
$$

Now, the determinant $\left|w_{m}\left(\frac{k}{2^{n}}\right)\right|, 0 \leq k<2^{n}, 0 \leq m<2^{n}$ is of order $2^{n}$ and does not vanish. Thus the numbers $w_{m}\left(\frac{k}{2^{n}}\right)$ are linearly independent and 
it follows from (3.10) that

$$
\Gamma_{n}^{(k)}=0 \quad, 0 \leq k<2^{n}
$$

Suppose that (3.9) is satisfied for all $m \in N$ and let $G(x)$ denote the continuous function $G(x)=\int_{0}^{x} f d \mu_{F}$. Using (3.11), we get

$$
G\left(\frac{2 k^{*}+1}{3^{n}}\right)=G\left(\frac{2 k^{*}}{3^{n}}\right) \quad, 0 \leq k<2^{n}
$$

and so $G(x)$ is a constant on $C$. It follows that $f$ is equivalent to zero on the Cantor set $C$. Thus the proof is completed.

Let $f$ be an absolutely integrable function on the interval $[0,1]$. The $\Psi$ - Fourier series of $f$ with respect to the system $\left\{\psi_{n}, n \in N\right\}$ is defined by $S f=\sum_{n=0}^{\infty} a_{n} \psi_{n}$ where $a_{n}=\int_{0}^{1} f(x) \psi_{n}(x) f \mu_{F}$. The $n$-th partial sums of this series will be denoted by $S_{n} f=\sum_{k=0}^{n-1} a_{k} \psi_{k}, n \in N$. Notice for $n \in P$ and $x \in[0,1]$

$$
\left(S_{n} f\right)(x)=\sum_{k=0}^{n-1}\left(\int_{0}^{1} f(t) \psi_{k}(t) d \mu_{F}(t)\right) \psi_{k}(x)=\int_{0}^{1} f(t) D_{n}(x, t) d \mu_{F}(t)
$$

where

$$
D_{n}(x, t)=\sum_{k=0}^{n-1} \psi_{k}(x) \psi_{k}(t)
$$

denote to the $n$-th Dirichlet kernel.

(1) $D_{2^{n}}(x, t)=\prod_{i=0}^{n-1}\left(1+\varphi_{1}(x) \varphi_{i}(t)\right)$

(ii) $D_{2^{n}}(x, t)=2^{n} x_{J_{2^{n}+k}}^{(t)} \quad, x \in J_{2^{n}+k} \quad$ for some $k, 0 \leq k<2^{n}$

PRoOF. Let $n \in N$ and $x, t \in C \backslash C^{*}$. (i) Using (1.7), (3.6), and (3.12), we have

$$
\begin{aligned}
D_{2^{n}}(x, t) & =\sum_{1=0}^{2^{n}-1} \psi_{i}(x) \psi_{i}(t)=\sum_{1=0}^{2^{n}-1} w_{i}(\xi(x)) w_{i}(\xi(t))=\sum_{i=0}^{2^{n}-1} w_{i}(\xi(x)+\xi(t)) \\
& =\prod_{i=0}^{n-1}\left(1+r_{1}(\xi(x)) r_{1}(\xi(t))\right)=\prod_{i=0}^{n-1}\left(1+\varphi_{1}(x) \varphi_{1}(t)\right) .
\end{aligned}
$$

(11) Fix $k \in\left\{0,1, \ldots, 2^{n}-1\right\}$, and let $x \in J_{2^{n}+k}=\left[\frac{2 k^{*}}{3^{n}}, \frac{2 k^{*}+1}{3^{n}}\right)$, then $\xi(x) \in I_{n}\left(k \cdot 2^{-n}\right)$. Using the properties of the mapping $\xi$ and the Dirichlet kernel $D_{2} n$ in the Walsh case, we get

$$
D_{2^{n}}(x, t)=\sum_{i=0}^{2^{n}-1} w_{i}(\xi(x)+\xi(t))=2^{n} x_{I_{n}\left(k \cdot 2^{-n}\right)}(\xi(t))=2^{n} x_{J_{2^{n}+k}}(t) .
$$

THEOREM 4. Let $f$ be a continuous function on $C \backslash C^{*}$, then $S_{2^{n}} f$ converges uniformly to $f$.

PROOF. For every $x \in C \backslash C^{*}$ and $n \in N$, we define $\alpha_{n}=\alpha_{n}(x)$ and $\beta_{n}=\beta_{n}(x)$ by $\alpha_{n}=\frac{2 k^{*}}{3^{n}} \leq x<\frac{2 k^{*}+1}{3^{n}}=\beta_{n}$ for some $k, 0 \leq k<2^{n}$ where $k^{*}$ is defined in (2.2). By the canonical map $\xi$ the set $\left(C \backslash c^{*}\right) \cap\left(\alpha_{n}, \beta_{n}\right)$ maps onto the dyadic interval $I_{n}\left(k \cdot 2^{-n}\right)$ and $\mu_{F}\left(\left[\alpha_{n}, \beta_{n}\right)\right)=2^{-n}$. Then the 
function $f$ can be written as

Using corollary 3

$$
f(x)=2^{n} \int_{n}^{\beta} f(x) d \mu_{F}(t)
$$

$$
\begin{aligned}
\left(S_{2^{n}} f\right)(x) & =\int_{0}^{1} f(t) D_{2^{n}}(x, t) d \mu_{F}(t)=2^{n} \int_{0}^{1} f(t) x_{\left[\alpha_{n}, \beta_{n}\right)} d \mu_{F}(t) \\
& =2^{n} \int_{\alpha_{n}}^{\beta_{n}} f(t) d \mu_{F}
\end{aligned}
$$

From (3.13) and since $f$ is continuous, 1.e $\forall \varepsilon>0 \exists \delta>0$ :

$$
\begin{aligned}
&|f(t)-f(x)|<\varepsilon \text { whenever }|t-x|<\delta, x, t \in\left[\alpha_{n}, \beta_{n}\right) \text {. Therefore, } \\
& \qquad\left(S_{2^{n}} f\right)(x)-f(x) \mid=\left|2^{n} \int_{n}^{\beta_{n}}(f(t)-f(x)) d \mu_{F}(t)\right| \\
& \leq 2^{n} \int_{n}^{\beta}|f(t)-f(x)| d \mu_{F}(t)<\varepsilon
\end{aligned}
$$

and the proof is completed.

\section{THE CANTOR SET UITH CONSTANT DISSECTION RATIO}

The constructed system $\Phi$ in (3.1) can be describe on any Cantor set with constant dissection ratio. The generating intervals of the Cantor set of general ratio $p / q, 0<p<q, p, q \in P$ are defined by

$$
J_{2^{n}+k}^{p / q}=\left[\frac{\lambda_{2^{n}+k}-(q-p)^{n}}{(2 q)^{n}}, \frac{\lambda_{2}^{n}+k}{(2 q)^{n}}\right] \quad, 0 \leq k<2^{n}
$$

where $\lambda_{1}$ are determined by the recursive formula

$$
\lambda_{1}=1 \text { and } \lambda_{2^{n}+k}=(q-p)^{1-k} \lambda_{2^{n-1}+k}+k_{n-1}(q+p)(2 q)^{n-1}
$$

$k_{1}$ being the dyadic coefficients of $k$ in $(1.1)$. It is easy to see that $\lambda_{2} n=2^{n}$ for each $n \in N$. Then the Cantor set $c^{p / q}$ of ratio $p / q$ is

$$
C^{p / q}=\bigcap_{n=0}^{\infty} U_{k=0}^{2^{n}-1} J_{2^{n}+k}^{p / q}
$$

$c^{p / q}$ contains the end-points of each closed interval $J_{p / q}^{p / q}$. The closed intervals $J_{m}^{p / q}=\left[\alpha_{m}, \beta_{m}\right]$ satisfies the following four properties:

$$
\begin{aligned}
& \int_{2^{n+1}+2 k+8}^{p / q}<\int_{2^{n+k}}^{p / q} \quad, s=0,1 \\
& \text { (ii) } J_{2^{n+1}+2 k}^{p / q} \cap J_{2^{n+1}+2 k+1}^{p / q}=\phi \\
& \alpha_{2^{n}+k}=\alpha_{2^{n+1}+2 k}, \quad \beta_{2^{n}+k}=\beta_{2^{n+1}+2 k+1} \\
& \text { for } 0 \leq k<2^{n}, n \in N \text {, and }
\end{aligned}
$$

$$
\lim _{n \rightarrow \infty} \max _{0 \leq k<2}\left|J_{2^{n}+k}^{p / q}\right|=0
$$

The open intervals removed in this construction are defined by

$E^{p / q}=\bigcup_{n=0}^{\infty} U_{k=0}^{2^{n}-1} E_{2^{n}+k}^{p / q}$ where $E_{2^{n}+k}^{p / q}=\left(\frac{\lambda_{2}^{n+1}+2 k}{(2 q)^{n+1}}, \frac{\lambda_{2^{n+1}+2 k+1}-(q-p)^{n+1}}{(2 q)^{n+1}}\right)$ For each $n \in N$, the class $A_{n}^{p / q}=\left\{J_{2^{n}+k}^{p / q}: 0 \leq k<2^{n}\right\}$, contains $2^{n}$ closed

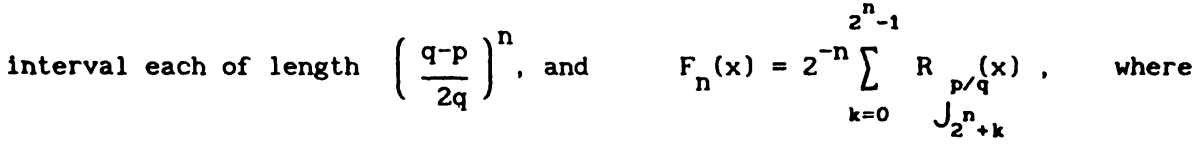




$$
R_{\substack{p / q \\ J_{2}^{n}+k}}(x)=\left(\frac{2 q}{q-p}\right)^{n} \int_{0}^{x} x_{\substack{j / q \\ J_{2}^{n}+k}}(t) d t
$$

$$
= \begin{cases}0 & x<\frac{\lambda_{2^{n}+k}-(q-p)^{n}}{(2 q)^{n}} \\ \left(\frac{2 q}{q-p}\right)^{n} \times-\frac{\lambda^{n}+k}{(q-p)^{n}}+1 & , x \in J_{2^{n}+k}^{\lambda_{2}^{n}+k} \\ 1 & , x>\frac{\lambda_{2}^{n}+k}{(2 q)^{n}}\end{cases}
$$

Hence,

$$
F_{n}(x)= \begin{cases}2^{-n}\left[\left(\frac{2 q}{q-p}\right)^{n} x-\frac{\lambda}{(q-p)^{n}}+1+k\right], & x \in \int_{2^{n}+k}^{p / q}, 0 \leq k<2^{n} \\ \frac{2 k+1}{2^{m}} & , x \in E_{2^{m-1}+k}^{p / q}, 0 \leq k<2^{m-1}, 1 \leq m \leq n\end{cases}
$$

Observe by properties (1), (11), and (1ii) above that $F_{n}$ is continuous and non-decreasing on $[0,1]$. In fact, each $F_{n}$ is linear on the intervals $\int_{2^{n}+k}^{p / q}$ and constant on each component of the set $[0,1] /\left(U_{k=0}^{2} \bigcup_{2^{n}+k}^{p / q}\right)$. The Cantor function associated with the Cantor set $c^{p / q}$ is

$$
F(x)=\lim _{n \rightarrow \infty} F_{n}(x)=\frac{2 k+1}{2^{m}}, x \in E_{2^{m-1}+k}^{p / q}, 0 \leq k<2^{m-1}, 1 \leq m<\infty
$$

THEOREM 5. If $\frac{1}{3} \leq \frac{p}{q}<1$, then there is a continuous strictly increasing function $L$ on $[0,1]$ such that $L\left(C^{p / q}\right)=C$.

PROOF. For each $n \in N, 0 \leq k<2^{n}$, we have

$$
\left|J_{2^{n}+k}^{p / q}\right|=\left(\frac{q-p}{2 q}\right)^{n}=\frac{\gamma}{3^{n}} \text { where } \quad \gamma=\left(\frac{3(q-p)}{2 q}\right)^{n}
$$

Since $\frac{1}{3} \leq \frac{p}{q}<1$, then $0 \leq \gamma<1$. Let $L_{n}$ be the monotone-increasing piecewise linear function, $L_{n}:[0,1] \rightarrow[0,1]$, mapping the end-points of $\int_{p / q}^{p / q}$ onto those of $J^{2^{n}+k} 2^{n}+k$ $0 \leq k<2^{n}$. Then for $n>m, L_{n}$ and $L_{m}$ differ only on $j_{2^{m}+k}^{p / q}, 0 \leq k<2^{m}$, in fact, $\left|L_{n}-L_{m}\right| \leq\left|J_{2 m+k}\right|=\frac{1}{3^{m}}$. So, for each $x$, the sequence $\left\langle L_{m}(x)\right\rangle$ converges and defines a function $L(x)$ on $[0,1]$. As $\left|L_{m}(x)-L(x)\right|=\lim _{n \rightarrow \infty}\left|L_{n}(x)-L_{m}(x)\right| \leq \frac{1}{3^{m}}$. $\left\langle L_{m}\right\rangle$ converges uniformly to $L[9]$. So $L$ is continuous and is clearly monotone increasing. We have $L([0,1])=[0,1]$ and

$$
L\left(J_{2^{n}+k}^{p / q}\right)=J_{2^{n}+k} \quad \text { for each } n \text { and } k, \quad 0 \leq k<2^{n} \text {. }
$$

so $L\left(C^{p / q}\right)=C$. We need only to show that $L$ is one-to-one. Suppose $(x, y) \subset[0,1]$. If either $x$ or $y$ lies in removed interval it is easy to see that $\quad L(y)>L(x)$. So suppose, $x$ and $y$ are in $c^{p / q} ;$ then as $c^{p / q}$ is nowhere dense there is an interval $\int_{2^{n}+k}^{p / q} s(x, y)$ for some $n, k$. But then $L(y)-L(x) \geq \frac{1}{3^{n}}$, so $L$ is strictly increasing and the proof is completed.

According to theorem 5, for each $n \in N, 0 \leq k<2^{n}$, and $\frac{1}{3} \leq \frac{p}{q}<1$.

$$
L\left(\frac{\lambda_{2^{n}+k}-(q-p)^{n}}{(2 q)^{n}}\right)=\frac{2 k}{3^{n}} \text { and } L\left(\frac{\lambda_{2^{n}+k}}{(2 q)^{n}}\right)=\frac{2 k^{*}+1}{3^{n}} \text {. }
$$


Now, the constructed system $\Phi$ in (3.1) can be generalized for any Cantor set $C^{p / q}$ in the following way : for each $x \in(0,1), p, q \in P$ and $n \in N$, $0 \leq k<2^{n+1}$, 1.e. $k=\sum_{1=0}^{n} k_{1} 2^{i}$, define

$$
\varphi_{n}(x)= \begin{cases}(-1)^{k_{0}}, & x \in J_{2^{n+1}+k}^{p / q} \\ 0 & \text { otherwise }\end{cases}
$$

The product system $\Psi$ is defined as in (3.4), it is orthonormal and complete system with respect to the measure $\mu_{F}$ on the general Cantor set $c^{p / q}$. Using theorem 5 and the mapping $L$, the results obtained in section 3 , for the ternary Cantor set $C$ can be easily generalized for the case $C^{p / q}$.

\section{REFERENCES}

1. ALEXITS, G. Convergence Problems of Orthogonal Series, Pergamon Press, New York - Oxford - Paris, 1961.

2. CHEN, C.F., and HSIAO, C.H. Time - Domain Synthesis Via Walsh Functions, Proc. IEE 122 (1975), 565-570.

3. CHEN, C.F., and HSIAO, C.H. Design of Piecewise Constant Gains for Optimal Control Via Walsh Functions, IEEE Trans. Autom. Control, AC-20 (1975), 596-602.

4. CHEN, C.F., and HSIAO, C.H. A State Space Approach to Walsh Series Solution of Linear Systems, Int. J. Syst. Sc1. $\underline{6}$ (1975), 833-858.

5. CHEN, W.L., and SHIH, Y.P. Analysis and Optimal Control of Time Varying Linear Systems Via Walsh Functions, Int. J. Control, 27, No.6 (1978), 917-932.

6. CHEN,W.L., and SHIH,Y.P. Shift Walsh Matrix and Delay differential Equations, IEEE Trans. Autom. Control, AC-23 (1978), 1023-1028.

7. CHEN, W.L. Walsh Series Analysis of Multi - Delay Systems, J. Franklin Inst. 313 (1982), 207-217.

8. CORRINGTON, M.S. Solution of Differential and Integral Equations with Walsh Functions, IEEE Trans. Circuit Theory CT-20 (1973), 470-476.

9. DEBARRA, G. Introduction to Measure Theory, Van Nostrand Reinhold press, New York - London, 1975.

10. FINE, N.J. On The Walsh Functions, Trans. Amer. Math. Soc. 65 (1949), 372-414.

11. PALEY, R.E.A.C. A Remarkable Series of Orthogonal Functions, Proc. Lond. Math. Soc. 34 (1932), 241-279.

12. Rademacher, H. Einige Sätze über Relhen von Allgemeinen Orthogonalen Funktionen, Math. Ann. 87 (1922), 122-138.

13. SCHIPP, F., WADE, W., and PETER, S. Walsh Series - An Introduction to the Dyadic Harmonic Analysis, Adam Hilger, England, 1990. 
14. TZAFESTAS, S.G. Walsh Series Approach to Lumped and Distributed System Identification. J. Franklin Inst. 305 (1978), 199-220.

15. WADE, H. R. Recent Developments in the Theory of Walsh Serles, Internat. J. Math. \& Math. Scl. 5. No.4 (1982), 625-673.

16. WALSH, J.L. A Closed Set of Normal Orthogonal Functions, Amer. J. Math, 45 (1923), 5-24. 


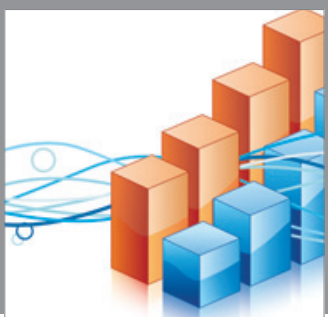

Advances in

Operations Research

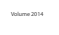

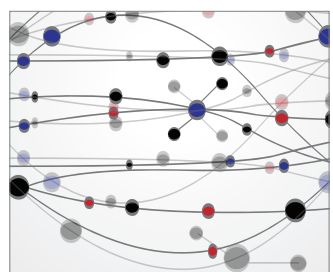

\section{The Scientific} World Journal
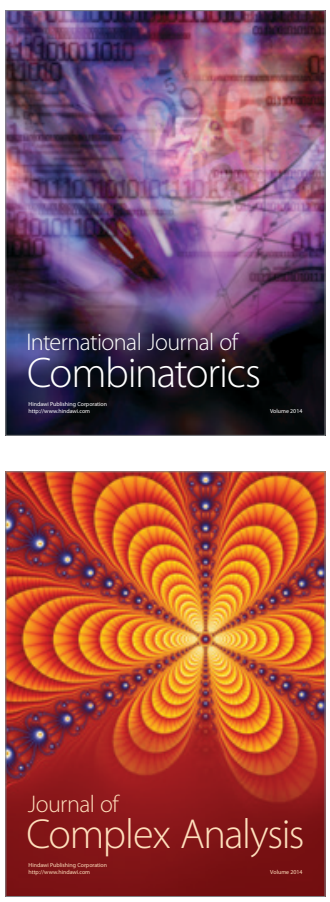

International Journal of

Mathematics and

Mathematical

Sciences
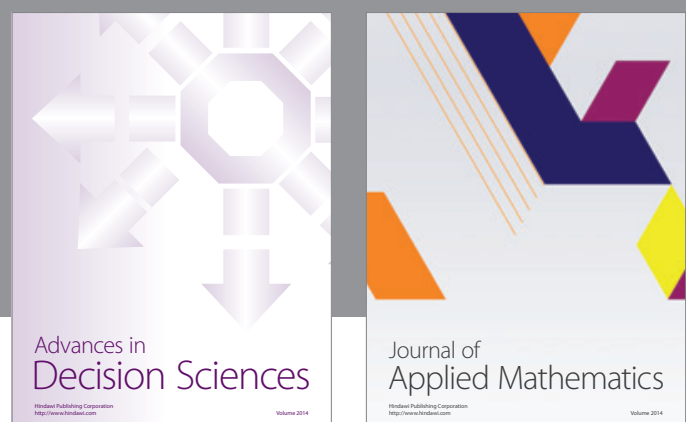

Journal of

Applied Mathematics
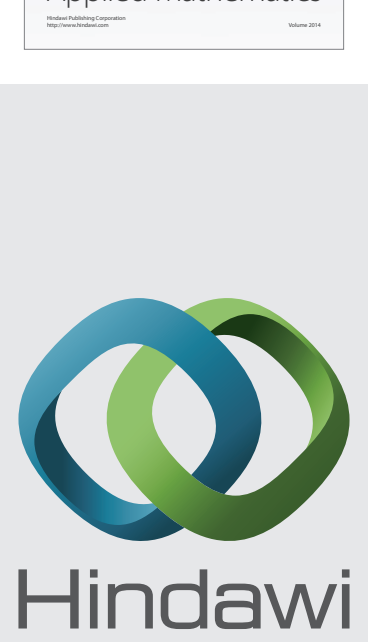

Submit your manuscripts at http://www.hindawi.com
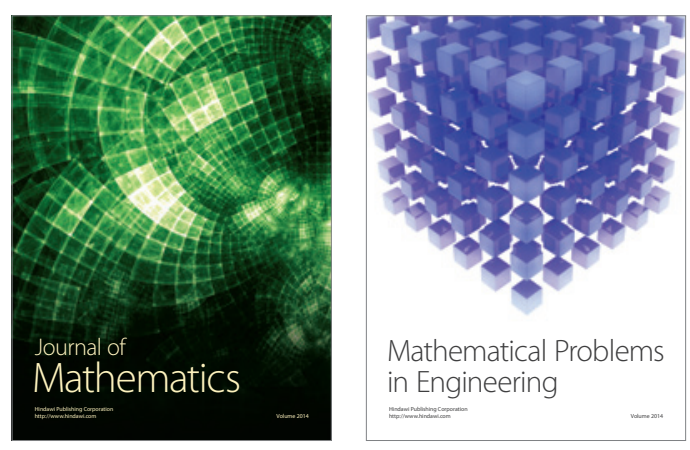

Mathematical Problems in Engineering
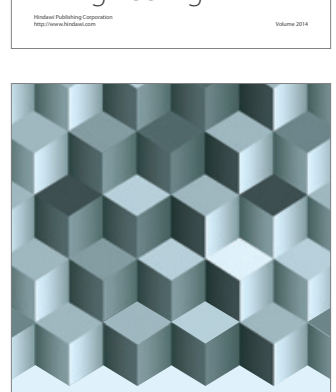

Journal of

Function Spaces
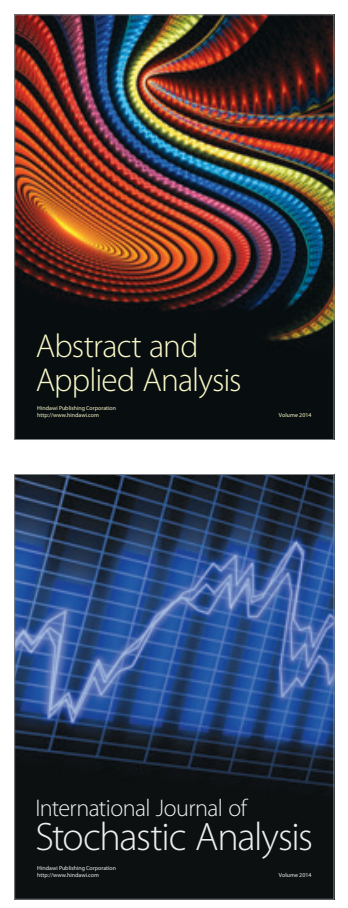

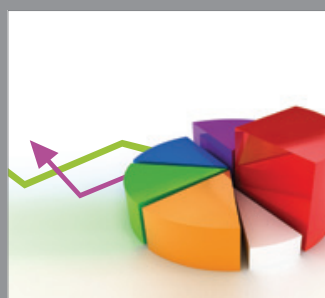

ournal of

Probability and Statistics

Promensencen
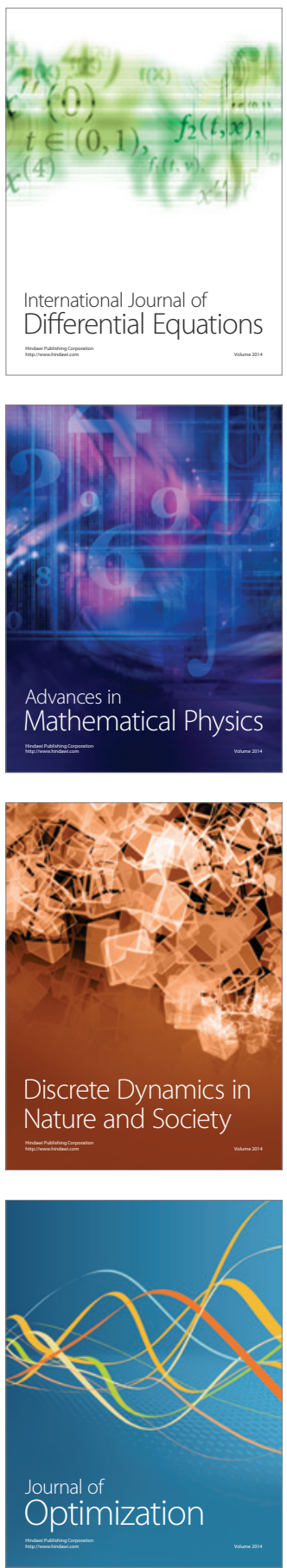